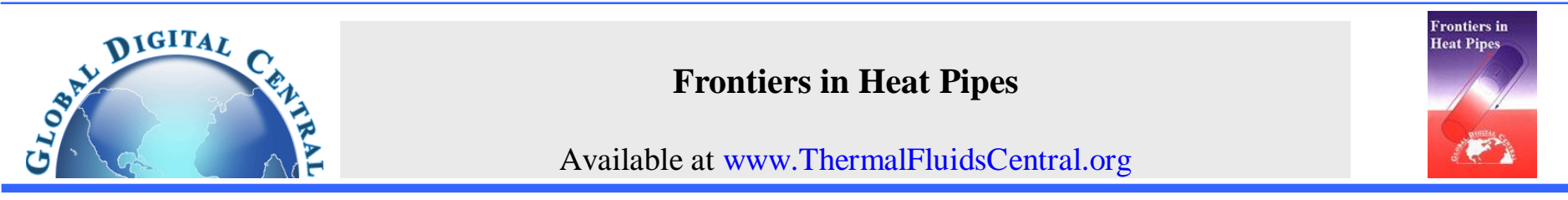

\title{
A STATISTICAL ANALYSIS OF TEMPERATURE OSCILLATIONS ON A FLAT-PLATE OSCILLATING HEAT PIPE WITH TESLA-TYPE CHECK VALVES
}

\author{
S.M. Thompson, H.B. Ma* \\ Department of Mechanical \& Aerospace Engineering, University of Missouri, Columbia, MO, 65211, USA
}

\begin{abstract}
Steady-state, oscillatory temperature signals occurring on the wall of two flat-plate oscillating heat pipes (FP-OHP) - one with Tesla-type check valves (TV FP-OHP) and another without - were analyzed using statistical methods in order to quantify and determine oscillating behavior associated with flow rectification. Using the Expectation-Maximization algorithm, a probability density function (PDF) governing the temperature signal was found to be multi-Gaussian and dependent on heat input and heat pipe region for both FP-OHPs investigated. Results further indicate that the inherent flow rectification within the TV FP-OHP provides for an evaporator temperature signal with a less heat-dependent, higher-kurtosis PDF and, at higher heat inputs, a higher: Pearson correlation coefficient and Shannon entropy. These results provide a unique means to quantify the pseudo-random feature of oscillatory temperature occurring on an OHP.
\end{abstract}

Keywords: Pulsating Heat Pipe, Gaussian Mixture Model, Shannon Entropy, Flow Rectification, Signal Analysis

\section{INTRODUCTION}

The oscillating (or pulsating) heat pipe (OHP), first introduced by Akachi (1990), is a two-phase heat transfer device which, unlike conventional heat pipes, contains no wick, has less operating limitations and may exist in a variety of form factors. It typically exists as either a serpentine-arranged capillary tube - a tubular OHP (T-OHP) - or a milled, serpentine channel on a flat medium - a flat-plate oscillating heat pipe (FP-OHP). The OHP is partially filled with a working fluid and then sealed. The amount of working fluid used is quantified via the filling ratio which is defined as the volume of liquid over the total volume of the OHP channel structure. The channel hydraulic diameter is made sufficiently small to allow surface tension forces to dominate, i.e.:

$$
D_{H} \lesssim 2 \sqrt{\frac{\gamma}{g\left(\rho_{l}-\rho_{v}\right)}}
$$

During operation, non-uniform vapor volume expansion in the evaporator coupled with condensation in the condenser gives rise to a highly complex, unsteady pressure field that varies along the channel length. For a functional OHP, this thermally-driven pressure field results in pseudo-chaotic displacement/circulation (net displacement) of the internal working fluid. The displacement of the working fluid is highly oscillatory with bulk circulation occurring at a much lower frequency. The bulk circulation is beneficial as it results in more liquid being pumped through the evaporator therefore increasing convection heat transfer rates and decreasing the overall thermal resistance.

A minimal heat input (activation heat input), or temperature difference, is required to start and sustain OHP operation. Upon startup of an OHP, the internal fluid begins to displace chaotically, the mean temperature difference between the evaporator and condenser decreases and the surface temperature field begins to oscillate with time. At relatively high heat inputs, the OHP may stop operating due to a condition referred to as 'dry-out' (Cai et al., 2006; Kammuang-Lue et al., 2009; Khandekar et al., 2003; Yang et al. 2008). At dry-out, the OHP is unable to replenish the evaporator with liquid in order to sustain operation.

Many qualitative assessments have been made on the internal, two-phase flow patterns occurring within an operating OHP. Khandekar et al. (2009) experimentally investigated a copper, singleloop T-OHP with glass adiabatic section charged with ethanol at a filling ratio of 0.6. At a constant heat input of $20 \mathrm{~W}$, distinct operating states were observed to occur throughout time. These operating states were characterized by their unique flow patterns, pressure/temperature variations and the corresponding thermal resistance. The operating state that corresponded with optimal thermal performance consisted of dominant vapor presence in the evaporator and dominant liquid presence in the condenser and the fluid oscillating steadily with time. Circulatory flow was observed, but the flow direction was noted to change with experimental trials. Non-oscillatory operating states were also observed where the liquid remained static in the condenser resulting in evaporator temperature rises. Tong et al. (2001) visualized the flow patterns within a closed-looped, glass T-OHP using a charged coupled device. The T-OHP was charged with methanol at a filling ratio of 0.6 and the heat input was held constant at $50 \mathrm{~W}$. It was found that the liquid and vapor were unevenly distributed along the tube length and that the fluid oscillated and circulated during steady-state OHP operation. The circulatory flow direction changed with repeated experimental trials and the circulation velocity increased with heat input. $\mathrm{Xu}$ et al. (2005) visualized internal fluid motion within a capillary glass tube OHP with a high speed camera. The OHP was charged with either methanol or water both at a filling ratio of 0.7 . Two heat inputs were investigated: $10 \mathrm{~W}$ and $30 \mathrm{~W}$. Flow circulation was

\footnotetext{
*Corresponding author.Email: MaH@missouri.edu
} 
observed to accompany the highly-oscillatory flow and the circulatory direction changed with time. During higher heat inputs, the displacement amplitude of the working fluid increased and fluid oscillations became more severe and random.

Neutron radiography has also been utilized to observe internal fluid motion directly through metallic walled OHPs. Wilson et al. (2008) investigated tubular copper T-OHPs filled with either water or water-diamond nanofluid with filling ratios of 0.50 . It was found that at low heat inputs, the internal fluid oscillations were intermittent and random, but at higher heat inputs more stable and consistent fluid oscillations with higher velocities occurred. Circulatory behavior was observed to occur along with the oscillating motions of the internal working fluid. Thompson et al. $(2009 ; 2010)$ visually investigated a copper three-dimensional FP-OHP (3D FP-OHP) filled with acetone at a filling ration of 0.73 . Internal fluid oscillations were clearly observed upon start-up of the 3D FP-OHP and strong liquid/vapor segregation was noted to occur at high heat inputs.

The flow-reversing, stochastic-nature of internal fluid motion within an OHP can be rectified via utilization of check-valves (Akachi, 1990). With fewer reversals in flow direction, the thermal performance of the OHP increases. Consistent circulation is beneficial for heat transfer as it allows for increased forced convection and more timeaveraged liquid mass in the evaporator. Some have employed floatingball check-valves on tubular OHPs (Rittidech et al., 2007), as well as utilizing tubing with non-constant internal diameter (Liu et al., 2007) in order to rectify circulatory motion. For both design alterations, the OHP thermal resistance was found to decrease.

Recently, Tesla-type check valves were installed within an FPOHP for flow rectification (Thompson et al., 2011). The Tesla valve (Tesla, 1920) is a 'no-moving-parts' valve that connects in-parallel to a channel containing circulating fluid. The valve is capable of promoting net circulatory flow by creating a higher pressure drop in the backwardflowing direction mainly by jet interacting flow at the valve exits (Bardell, 2000). Using neutron radiography and image analysis techniques, it was confirmed that the Tesla-type check valves effectively rectified the internal flow within the FP-OHP and promoted a common, net circulatory direction of the working fluid with an accompanied $20 \%$ reduction in OHP thermal resistance.

Due to the hydro-thermal coupled OHP operation, analysis of the time-varying temperature field, or 'signals', provides insight into the internal fluid dynamics and the operating state of the OHP itself. Many unique methods have been employed to characterize the temperature signals - including the use of: peak-to-peak amplitudes (Thompson et al., 2011), power spectral density analysis (Xu and Zhang, 2005), autocorrelation functions and Kolmogorov entropy (Song and $\mathrm{Xu}$, 2009), recurrence plots and Lyapunov entropy (Qu et al., 2009), nonlinear auto-regressive moving average (NARMAX) models (Chen et al., 2009) and nonlinear autoregressive exogenous (NARX) models (Lee and Chang, 2009).

The current investigation employs statistical methods and parameters to uniquely characterize the temperature signals occurring on the surface of two flat-plate oscillating heat pipes - one with (regular FP-OHP) and one without Tesla-type check valves (TV FP-OHP). The main objectives were: 1) to estimate a probability density function (PDF) governing the 'sampling' of temperature on the surface of the regular FP-OHP and TV FP-OHP and 2) to expose any latent differences between the estimated PDF and temperature signals in order to thermally detect internal flow rectification caused by the Tesla-type check valves. These methods have the potential to eliminate the need for visual confirmation methods of flow rectification within an OHP which demand novel technology and image processing techniques.

\section{PROTOTYPE DEVELOPMENT}

Two flat-plate oscillating heat pipes were manufactured - one without (regular FP-OHP) and one with Tesla-type check valves (TV FP-OHP) (Thompson et al., 2011). Excluding the Tesla-type check valves, the designs for each heat pipe were exactly the same. Each FP-OHP base plate was made from electronic-grade copper and, as shown in Fig. 1, had overall dimensions of $136 \times 74.5 \times 2.75 \mathrm{~mm}^{3}$. Each design consisted of six arc-style turns in the evaporator and condenser. With the aid of Eq. (1), the closed-loop channel structure was designed to have a square cross-section with a hydraulic diameter of $1.5 \mathrm{~mm}$.

The Tesla-type check valves were installed in the predefined adiabatic section of the TV FP-OHP and, although designed mainly for clear visualization purposes, were previously found to be fully capable of rectifying and promoting circulatory flow during OHP operation (Thompson et al., 2011). For the current design, the preferential flow direction was counter clockwise (with regard to Fig. 1).

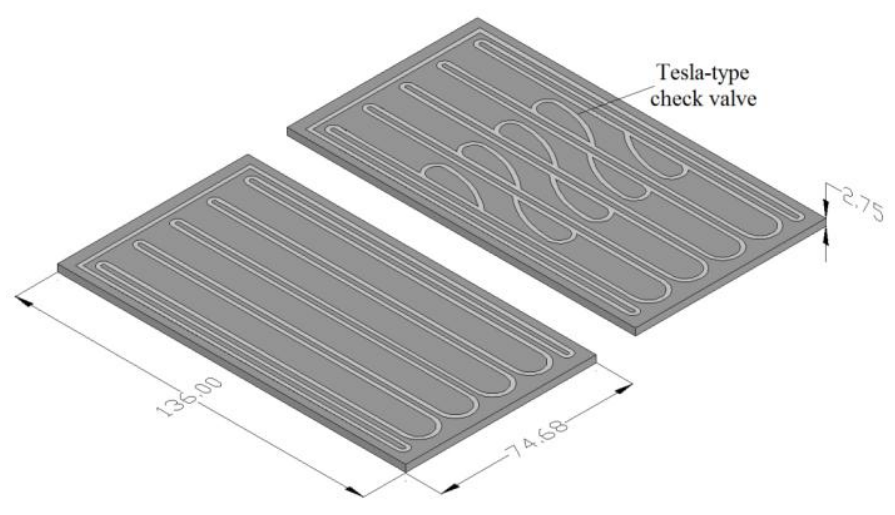

Fig. 1 Base plate for: regular FP-OHP (left) and TV FP-OHP (right) (units in millimeters)

Each base plate was sealed by brazing a thin copper cover plate $(0.25 \mathrm{~mm})$ onto its surface. A copper charging tube (1.58 mm OD) was soldered to the side of each sealed base plate and each device was evacuated via a vacuum pump. Both heat pipes were charged with high-chromatography water to a filling ratio of $0.70 \pm 0.01$. The charging tube was sufficiently crimped to maintain the internal vacuum pressure.

\section{EXPERIMENTAL SETUP AND PROCEDURE}

Surface temperatures on both the regular FP-OHP and TV FP-OHP were experimentally measured while providing a constant heat flux in the evaporator and constant-temperature in the condenser. The experiment was accomplished via the setup provided in Fig. 2.

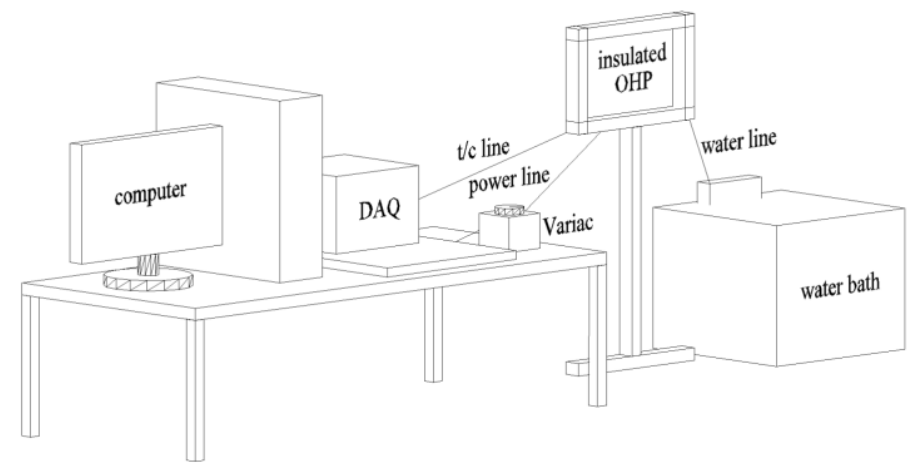

Fig. 2 Experimental setup

Each heat pipe was bottom-heated with equivalent heating and cooling lengths of $38 \mathrm{~mm}( \pm 0.5 \mathrm{~mm})$ as shown in Fig. 3. Constant temperature cooling was accomplished by firmly attaching two aluminum cooling blocks $\left(101.6 \times 38.1 \times 19 \mathrm{~mm}^{3}\right.$ each) to the condenser of each heat pipe. Each cooling block possessed two 
channels (8.73 mm ID) allowing for in-series flow of temperaturecontrolled water which was circulated and held at a constant temperature of $20^{\circ} \mathrm{C}\left( \pm 0.1{ }^{\circ} \mathrm{C}\right)$ using a Julabo $\mathrm{F} 34$ water bath. Constant, uniform heat input was applied to the evaporator using an aluminum heating block $\left(76.2 \times 38.1 \times 9.5 \mathrm{~mm}^{3}\right)$ with four $6.35 \mathrm{~mm}$ (OD) cartridge heaters (Watlow $150 \mathrm{~W}$ ) embedded within it. The heating block was firmly held against the cover plate side of evaporator surface using aluminum C-clamps. Thermal contact resistances were minimized using thermal paste (Omegatherm "201") which was applied between all cooling and heating surfaces.

Eight thermocouples (Type-T, $\pm 1^{\circ} \mathrm{C}$ ) - four in the evaporator $(\mathrm{T} 1$ - T4) and four in the condenser (T5 - T8) - were located along the nonheated side of each heat pipe as shown in Fig. 3. Each heat pipe, with attached heating and cooling blocks, was encapsulated with fiberglass insulation and held in the bottom-heated, vertical orientation in an aluminum test stand as shown in Fig. 2.

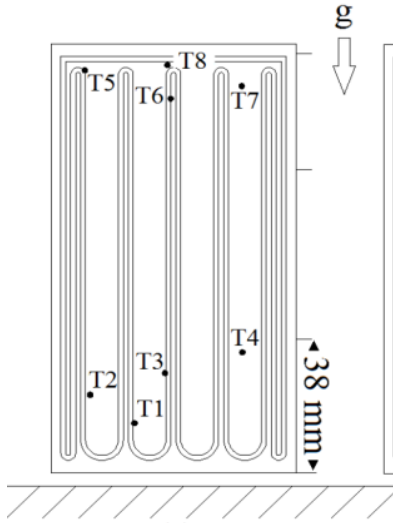

(a)

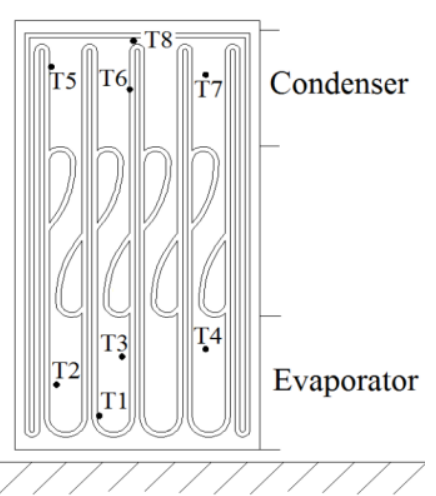

(b)
Fig. 3 Base plates with thermocouple locations and heating mode: (a) Regular FP-OHP, (b) TV FP-OHP

Temperatures at $\mathrm{T} 1-\mathrm{T} 8$ were collected at a sampling rate of 100 $\mathrm{Hz}$ for various heat inputs by using a data acquisition system (DAQ, National Instruments SCXI-1600). Power input to the cartridge heaters (in the heating block) was voltage-controlled via a variable autotransformer (Variac, $\pm 0.01 \mathrm{~W}$ ) and was varied in intervals ranging from $10 \mathrm{~W}$ to $20 \mathrm{~W}$. After a new power input was introduced, the OHP temperature field was allowed to achieve pseudo steady-state, whereupon the steady-state temperature field was recorded for approximately $3-5$ minutes. Heat losses were calculated to be approximately 2-3\% of power input based on measurements of the surface temperature of the insulated OHP system. Percent uncertainty in heat input was estimated to be approximately $1 \%$. For safety reasons, all experiments ceased when the maximum evaporator temperature exceeded $110{ }^{\circ} \mathrm{C}$. The described testing procedure and experimental setup were the same for both heat pipes investigated and each heat pipe was tested separately.

\section{ANALYSIS}

\subsection{Sample Data Preparation}

The recorded temperature signals (or arrays) were shifted to oscillate about zero by subtracting the mean temperature from each individual temperature, i.e.:

$$
T_{S, i, j}=T_{i, j}-\frac{1}{n} \sum_{i=1}^{n} T_{i, j}
$$

where $\mathrm{n}$ is the number of temperature data points collected at a single thermocouple location during steady-state for a given heat input and was fixed and held constant at 15,000 points - corresponding to the first 150 seconds of a steady-state temperature signal. A regional, shifted temperature array (RST), $\mathbf{T}_{\mathrm{RS}}$, was defined by concatenating the shifted temperature signals, $\mathbf{T}_{\mathrm{S}, \mathrm{j}}$, within each region. For example, the evaporator RST consisted of all sample data from $\mathbf{T}_{\mathrm{S}, 1}-\mathbf{T}_{\mathrm{S}, 4}$.

Histograms of the evaporator and condenser RST, with bin widths, w, were produced based on Freedman-Diaconis' rule (Freedman and Diaconis, 1981), i.e.:

$$
w=\frac{2 I Q R}{N^{1 / 3}}
$$

where IQR is the interquartile range which was found by ordering the RST data and finding the difference between the $75^{\text {th }}$ percentile and $25^{\text {th }}$ percentile, and $\mathrm{N}$ is the sample size of the RST which was exactly 60,000 .

\subsection{Probability Density Function Fitting}

For a given heat input, $\mathrm{q}$, the steady-state RST array, $\mathbf{T}_{\mathrm{RS}}$, in either the evaporator or condenser was regarded as recorded emissions from a random variable, $\Omega_{\mathrm{q}}$, which sampled from an unknown, continuous probability density function, $\mathrm{f}_{\mathrm{q}}$. The probability, $\mathrm{P}$, of $\Omega_{\mathrm{q}}$ being within the global minimum and maximum should then satisfy:

$$
\mathrm{P}\left[T_{R S, \text { min }} \leq \Omega_{q} \leq T_{R S, \text { max }}\right]=\int_{T_{R S, \text { min }}}^{T_{R S, \max }} f_{q} d T_{R S}=1
$$

In most cases, a basic form of $\mathrm{f}_{\mathrm{q}}$ must be assumed prior to the data fitting procedure. Since it was observed that the histograms representing the RST sample data possessed multimodal features, a PDF with varying degrees of modality was sought. A PDF used commonly for fitting sample data with inherent multimodality is the Gaussian mixture model (GMM). The GMM consists of the linear, weighted superposition of $\mathrm{M}$ unimodal Gaussian PDFs, or mixture components, i.e.:

$$
f_{q}\left(T_{R S, i} \mid \lambda\right) \approx \sum_{k=1}^{M} \pi_{k} f_{k}\left(T_{R S, i} \mid \lambda_{k}\right)
$$

with:

$$
f_{k}\left(T_{R S, i} \mid \lambda_{k}\right)=\frac{1}{\sqrt{2 \pi \hat{\sigma}_{k}^{2}}} \exp \frac{-\left(T_{R S, i}-\hat{\mu}_{k}\right)}{2 \hat{\sigma}_{k}^{2}}
$$

where $\hat{\mu}_{\mathrm{k}}$ is the unbiased estimator for the mean and $\hat{\sigma}_{\mathrm{k}}$ is the unbiased estimator for the standard deviation for the $\mathrm{k}^{\text {th }}$ mixture component's unimodal PDF, $\mathrm{f}_{\mathrm{k}}$, within the GMM, respectively. The weight for each mixture component, $\pi_{\mathrm{k}}$, is the approximate probability that $\mathrm{f}_{\mathrm{k}}$ will be selected within the GMM during the sampling process. Note that the sum of the $\mathrm{M}$ mixture component weights must sum to unity, i.e.:

$$
\sum_{k=1}^{M} \pi_{k}=1
$$

This idealized sampling process then consists of two steps. First, the $\mathrm{k}^{\text {th }}$ mixture component from the GMM is selected based on approximate probability, $\pi_{\mathrm{k}}$, then the final RST emission is selected from a unimodal Gaussian PDF, $\mathrm{f}_{\mathrm{k}}$. For a given GMM, the $\mathrm{M}$ x 3 parameter matrix, $\lambda$, is defined as:

$$
\lambda_{k}=\left[\pi_{k}, \hat{\mu}_{k}, \hat{\sigma}_{k}\right]
$$

Using MATLAB, the unknown GMM parameters were estimated using the Expectation-Maximization (EM) algorithm (Dempster et al., 1977). Through trial-and-error iteration, the EM-algorithm sought $\lambda$ that sufficiently minimized the negative of the log-likelihood function, as shown in Eq. (9):

$$
\theta=-\sum_{i=1}^{N} \ln \sum_{k=1}^{M} \pi_{k} f_{k}\left(T_{R S, i} \mid \lambda_{k}\right)
$$


For efficient convergence of the EM algorithm, the k-means clustering algorithm (Lloyd, 1982; Hartigan and Wong, 1979) was employed for parameter initialization (i.e.: initial guesses for component means, standard deviations and weights). Since the RST data were not multi-dimensional, no restrictions on the covariance were imposed during execution of EM-algorithm and the convergence criterion was set to $10 \mathrm{E}-5$.

A common challenge in estimating GMM parameters occurs when the true number of mixture components, $\mathrm{M}$, is not initially known. For the current analysis, the number of mixture components was estimated by successively executing the EM-algorithm for an assumed $M$ (typically ranging from $M=2$ to $M=40$ ). For each assumed $M$, the estimated GMM parameters were outputted and the accompanying Bayesian information criterion (BIC) was determined using:

$$
B I C=2 \theta+\Lambda \ln (N)
$$

where $\Lambda$ is the number of GMM parameters (i.e. number of terms in $\lambda$ ) found by:

$$
\Lambda=3 M
$$

The true number of mixture components was estimated as the $\mathrm{M}$ that provided for a global minimum in the BIC (Schwarz, 1978). This global minimum in BIC also corresponded to sufficient minimization in the negative of log-likelihood. This method conservatively predicted the number of mixture components in each GMM by penalizing overfitting PDFs that that used too many parameters.

For visualization purposes, the approximated PDF, $\mathrm{f}_{\mathrm{q}}$, was scaled so that it may be superimposed on the produced histograms:

$$
f_{q, \text { scaled }}=\frac{A_{\text {hist }}}{A_{f}} f_{q}
$$

where $A_{\text {hist }}$ was the area underneath the histogram and $A_{f}$ was the area underneath $\mathrm{f}_{\mathrm{q}}$. Both areas were found numerically in MATLAB.

\subsection{Other Extracted Parameters}

The evaporator and condenser RST and accompanying PDFs at various heat inputs were further quantified using the parameters presented in Eqs. (13) - (15). The magnitude of the sample Pearson product-moment correlation coefficient between evaporator and condenser RST, $\mathrm{r}_{\mathrm{e} / \mathrm{c}}$, was found using Eq. (13):

$$
r_{e / c}=\left|\frac{\sum_{i=1}^{N} T_{R S, e, i} T_{R S, c, i}}{\sqrt{\sum_{i=1}^{N} T_{R S, e, i}^{2}} \sqrt{\sum_{i=1}^{N} T_{R S, c, i}^{2}}}\right|
$$

The Shannon entropy, H, was found using Eq. (14). This particular form of entropy may be used to quantify the degree of uncertainty within a data string (Shannon, 1948).

$$
H=-\sum_{i=1}^{N} f_{q}\left(T_{R S, e, i}\right) \ln f_{q}\left(T_{R S, e, i}\right)
$$

The unbiased sample kurtosis, a statistical measure of the 'peakedness' in a PDF, was found for evaporator RST using Eq. (15).

$$
\left.K=\frac{(N-1)}{(N-2)(N-3)}(N+1) \frac{\frac{1}{N} \sum_{i=1}^{N} T_{R S, e, i}^{4}}{\left(\frac{1}{N} \sum_{i=1}^{N} T_{R S, e, i}^{2}\right)^{2}}-3(N-1)\right)+3
$$

\section{RESULTS}

\subsection{Multi-Component Probability Density Function}

The histograms representing the regional/shifted temperature (RST) in either the evaporator or condenser clearly exhibited multi-component characteristics at all heat inputs above OHP activation. This is demonstrated in Figs. 4-7 where the scaled PDF in either the evaporator or condenser, $\mathrm{f}_{\mathrm{q}, \text { scaled }}$, is superimposed with its respective RST histogram at heat inputs of $180 \mathrm{~W}$ and $240 \mathrm{~W}$ for both the regular FP-OHP and TV FP-OHP. Also included are the corresponding shifted, time-series temperature signals, $\mathbf{T}_{\mathrm{S}, \mathrm{j}}$. For conciseness, only the $\mathbf{T}_{\mathrm{S}, 3}$ and $\mathbf{T}_{\mathrm{S}, 6}$ temperature signals are shown, however, note that all other temperature signals in the respective regions had similar magnitudes and trends.
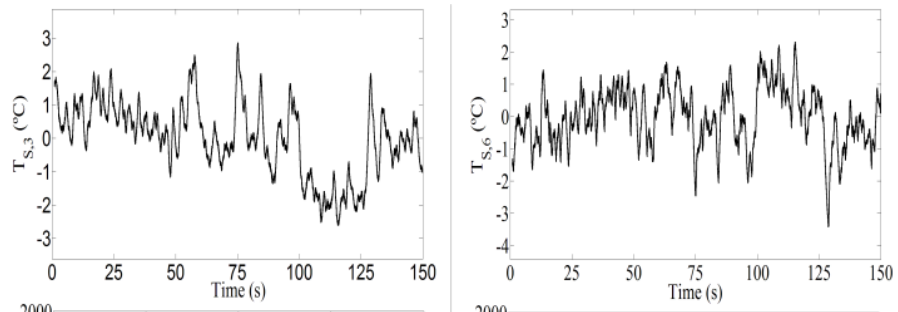

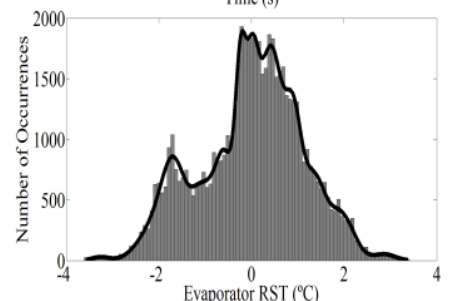

(a)

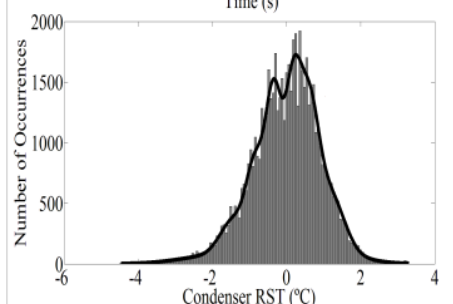

(b)
Fig. 4 TV FP-OHP: shifted time-series temperature signal and RST histogram superimposed with scaled PDF at a heat input of approximately $180 \mathrm{~W}$ for (a) evaporator (b) condenser
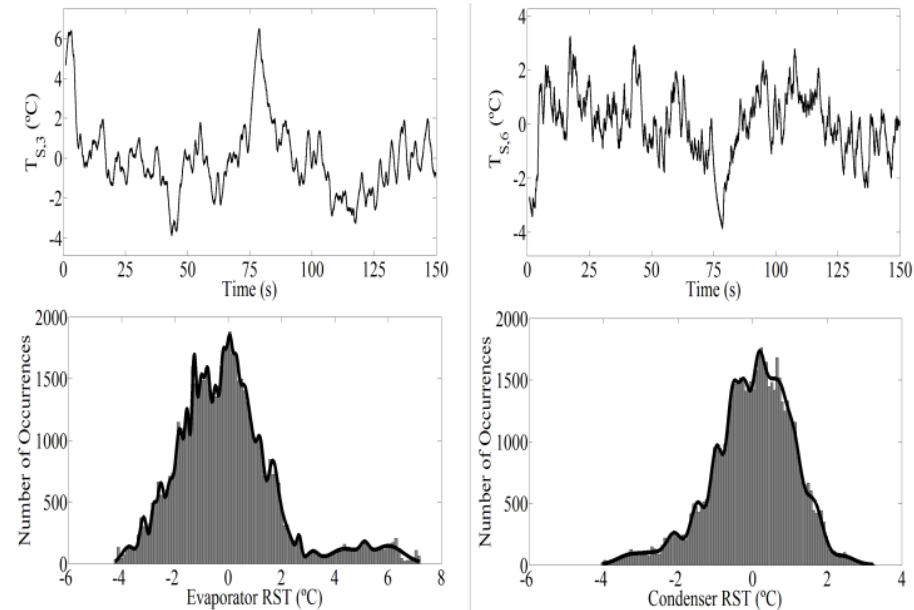

(a)

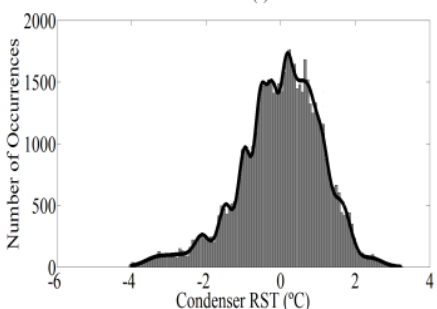

(b)

Fig. 5 Regular FP-OHP: shifted time-series temperature signal and RST histogram superimposed with scaled PDF at a heat input of approximately $180 \mathrm{~W}$ for (a) evaporator (b) condenser 

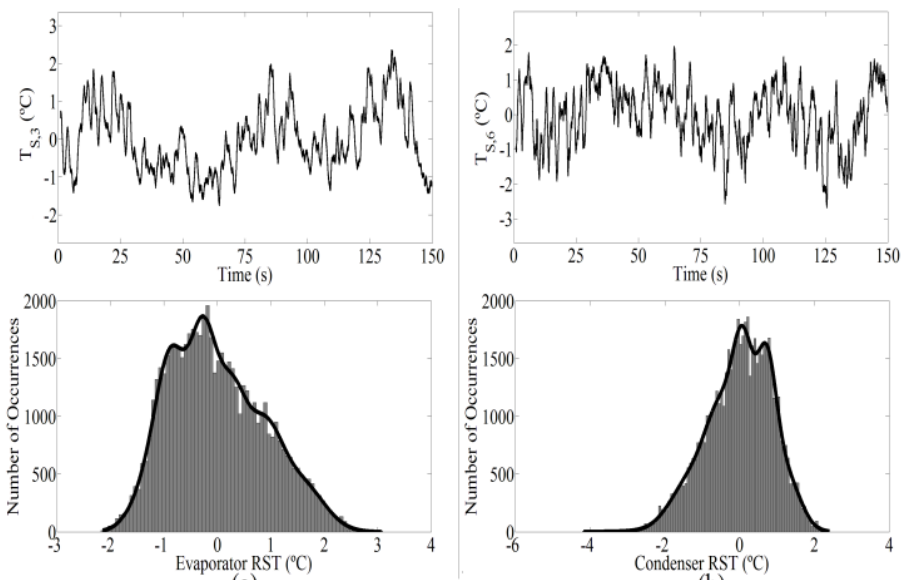

(a)

Fig. 6 TV FP-OHP: shifted time-series temperature signal and RST histogram superimposed with scaled PDF at a heat input of approximately $240 \mathrm{~W}$ for (a) evaporator (b) condenser
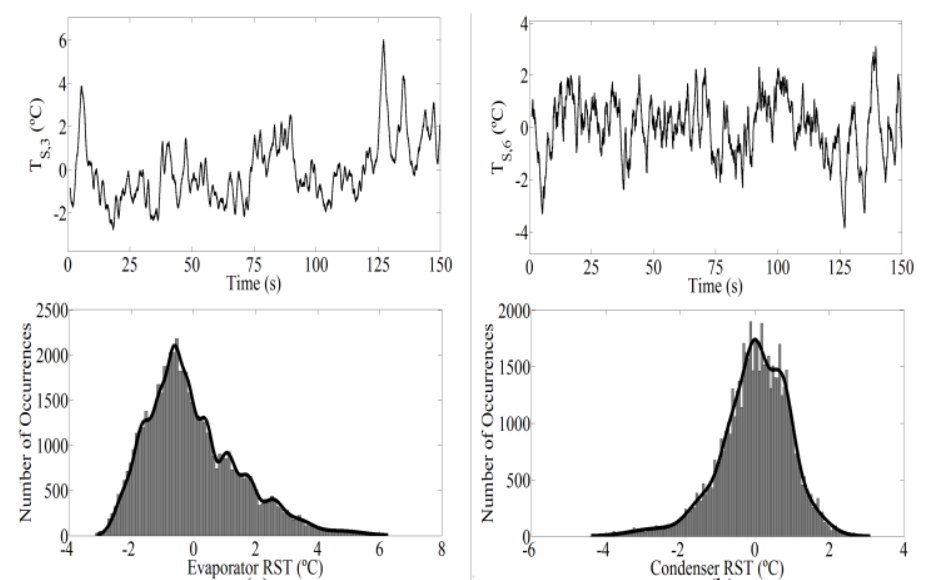

(a)

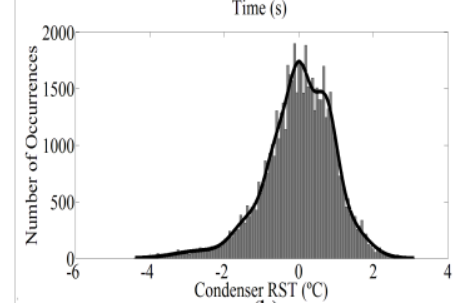

(b)

Fig. 7 Regular FP-OHP: shifted time-series temperature signal and RST histogram superimposed with scaled PDF at a heat input of approximately $240 \mathrm{~W}$ for (a) evaporator (b) condenser

From observing Figs. 4-7, it clear that the RST histograms and approximated PDFs demonstrated varying degrees of multimodality within the evaporator and condenser at various heat inputs for both the TV FP-OHP and regular FP-OHP. This multimodality is evident by the inherent asymmetry and distinct 'humps' within the provided Gaussianlike distributions. The assumption that the RST was multi-Gaussian (distributed via the GMM) appears very justifiable given the overlap of the estimated PDFs with the RST histograms. In general, the evaporator and condenser RST histograms for both the TV FP-OHP and regular FP-OHP were found to have the same qualitative features. The main difference was that the condenser RST histograms generally appeared to have a lesser degree of multimodality.

The temperature signals in both the evaporator and condenser, as shown in Figs. 4-7, were non-periodic and possessed time-varying amplitudes and this directly affected the degree of multimodality in the estimated PDFs. In general, the observed temperature signals consisted of both high-frequency and low-frequency components at all heat inputs above OHP activation. The high-frequency component may be attributed to sustenance of consistent fluid oscillations within the OHP. The low-frequency component, as evidenced by 'static' temperature rises in the evaporator and drops in the condenser, may be attributed to the temporary cessation of internal fluid motion occurring within the OHP. During these 'static' operating periods, heat is transferred from the evaporator to the condenser predominantly by conduction only. As heat input increases, the occurrence and duration of these temperature rises/drops decreases due to fluid motion within the OHPs becoming

more sustained. As shown in Figs. 5a - 5b, the occurrence of these static temperature rises/drops increases the range of the RST histogram and results in more-isolative and distinct mixture components. The low-frequency component of temperature signals also results in the formation of asymmetric tails in the RST histograms.

\subsection{Number of Mixture Components}

The number of mixture components in each multi-Gaussian PDF for evaporator and condenser RST, for both the TV FP-OHP and regular FP-OHP, is shown in Fig. $8-9$, respectively.

As seen from Figs 8 - 9, the number of mixture components in the PDF governing evaporator and condenser RST, $\mathrm{f}_{\mathrm{q}}$, was non-constant and varied with heat input. In general, the number of mixture components in either the evaporator or condenser RST was also different for both heat pipes at any given heat input. The estimated parameters, $\lambda$, were found to be different for all approximated PDFs even for PDFs with the same number of mixture components and this further indicates that the PDF changed with heat input.

Prior to OHP activation, the PDFs were found to be unimodal Gaussian and this was a result of the non-oscillating thermocouple signals consisting of only instrumental, Gaussian white noise. At heat inputs near but above the activation heat input $(\sim 80-100 \mathrm{~W})$; the multimodality in the evaporator and condenser PDFs became apparent due to the onset of temperature oscillations. During this heat input range (approximately $90-110 \mathrm{~W}$ ), the number of mixture components for each PDF was found to be very high (i.e. 30) and this was due to the infrequent occurrence of static temperature rises and drops in the evaporator and condenser, respectively. In general, as OHP operation (i.e. internal fluid oscillations) became more consistent during higher heat inputs (i.e. greater than $110 \mathrm{~W}$ ), the number of mixture components in the PDFs were found to be lower than those found during low heat input operation. As heat input increased from $110 \mathrm{~W}$ to $240 \mathrm{~W}$, the number of mixture components in the PDF governing condenser RST was found to generally increase for both heat pipes, while the number of mixture components in the PDF governing evaporator RST demonstrated less dependence on heat input. The number of mixture components in evaporator RST was generally higher than that in condenser RST. This may be attributed to the condenser being primarily filled with liquid and that a more time-varying twophase distribution exists within the evaporator during OHP operation (Thompson et al., 2011).

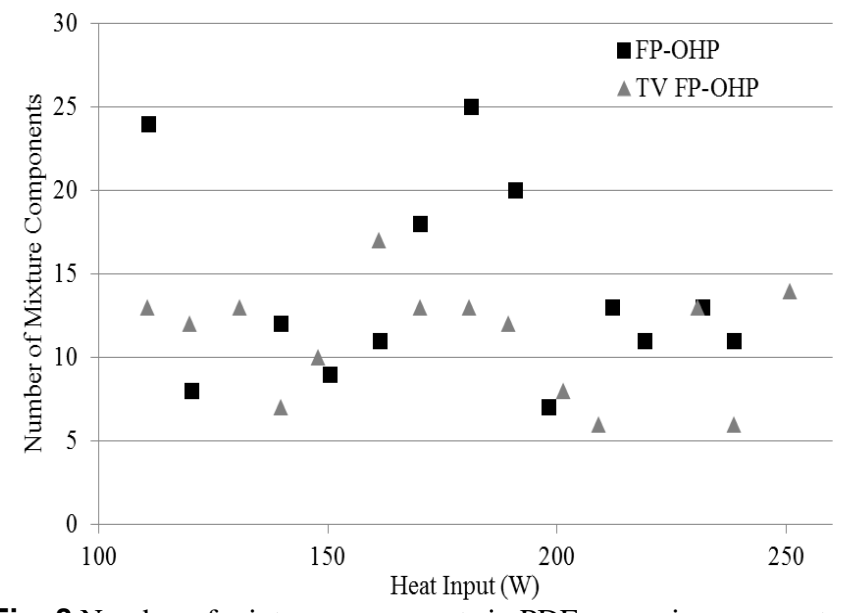

Fig. 8 Number of mixture components in PDF governing evaporator RST vs. heat input for regular FP-OHP and TV FP-OHP 


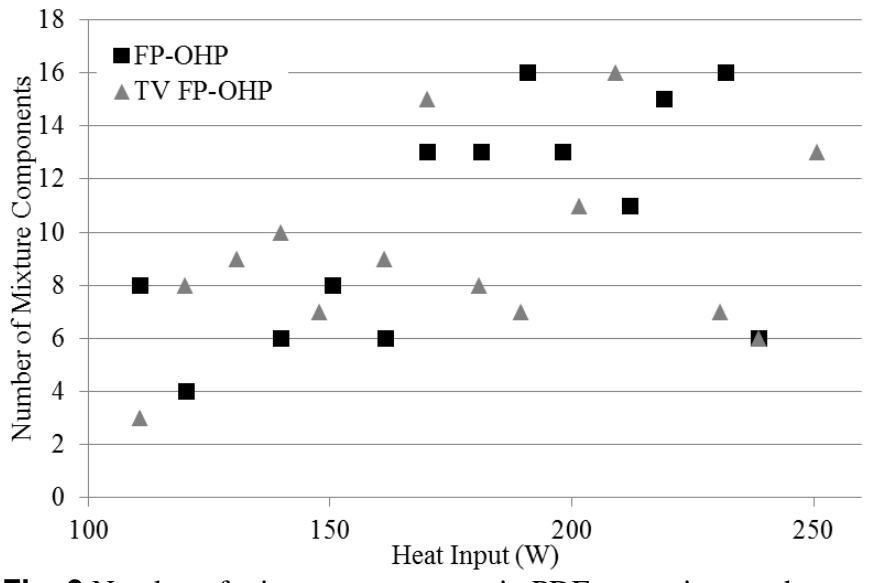

Fig. 9 Number of mixture components in PDF governing condenser RST vs. heat input for regular FP-OHP and TV FP-OHP

The number of mixture components in condenser RST was generally similar for both heat pipes, however, the regular FP-OHP typically provided for an evaporator RST with more mixture components. The overall variance in number of mixture components in evaporator RST for the TV FP-OHP was found to be less than that of the regular FP-OHP and this can be evidenced in Figs. 8 - 9. As shown in Fig. 8, the regular FP-OHP provided for an evaporator RST with a number of mixture components as high as 25 at a heat input of $180 \mathrm{~W}$. This relatively high value is mainly due to static temperature rises creating more isolated mixture components in the respective PDF. Note that since the PDF governing evaporator or condenser RST cannot be sufficiently modeled via a single Gaussian, it may be deduced that the oscillatory temperature field on the surface of an OHP consists of more than one frequency mode.

\subsection{Pearson Correlation Coefficient}

The temperature signals in the evaporator and condenser were found to be similar with one signal almost reflecting the other, i.e.: temperature rises in the evaporator corresponded to temperature drops in the condenser as shown in Figs. 4 - 7. The extent of this similarity was quantified using the Pearson correlation coefficient, $r_{\mathrm{e} / \mathrm{c}}$, as defined in Eq. (13). If the evaporator and condenser temperature signals were truly identical, both in magnitude and phase, then $r_{e / c}$ would be unity. The magnitude of the Pearson correlation coefficient between the evaporator and condenser RST for both the TV FP-OHP and regular FP-OHP at various heat inputs is provided in Fig. 10.

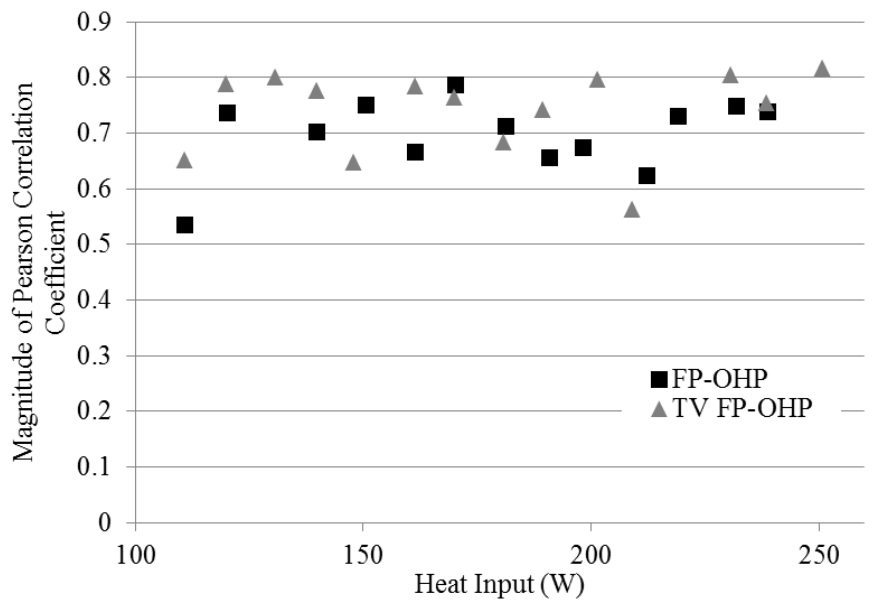

Fig. 10 Magnitude of Pearson correlation coefficient between evaporator and condenser RST vs. heat input for regular FPOHP and TV FP-OHP
It may be seen from Fig. 10, that there exists a non-unity Pearson correlation coefficient between the evaporator and condenser RST. This may be attributed to the temperature signals oscillating out of phase and to them having different amplitudes. In general, the Pearson correlation coefficient remained near-constant with heat input and was generally higher for the TV FP-OHP for most heat inputs. The TV FPOHP also provided for the maximum Pearson correlation coefficient of approximately 0.82 . Since the Pearson correlation coefficient had moderate magnitudes between evaporator and condenser RST typically between 0.6 and 0.8 - this confirms that limited similarity existed between the evaporator and condenser temperature signals.

\subsection{Shannon Entropy}

The Shannon entropy in evaporator RST at various heat inputs for both the regular FP-OHP and TV FP-OHP is provided in Fig. 11. From Fig. 11 , it is apparent that the TV FP-OHP provided for evaporator RST with less heat-dependent Shannon entropy that was consistently higher than that of the regular FP-OHP at heat inputs greater than approximately $170 \mathrm{~W}$. Since the Shannon entropy of a signal approaches zero with increasing predictability in future outcome, this indicates that the TV FP-OHP possesses a less-predictable evaporator temperature field with respect to time. This may be attributed to the TV FP-OHP having less static temperature rises and a more variant twophase distribution in its evaporator - both being a result of more frequent cross-channel circulation occurring in its evaporator. The higher Shannon entropy inherent in the TV FP-OHP evaporator temperature signals is further explained by the respective GMMs having more equal mixture component weights (i.e. more uniform $\pi$ ) as compared to those of the regular FP-OHP.

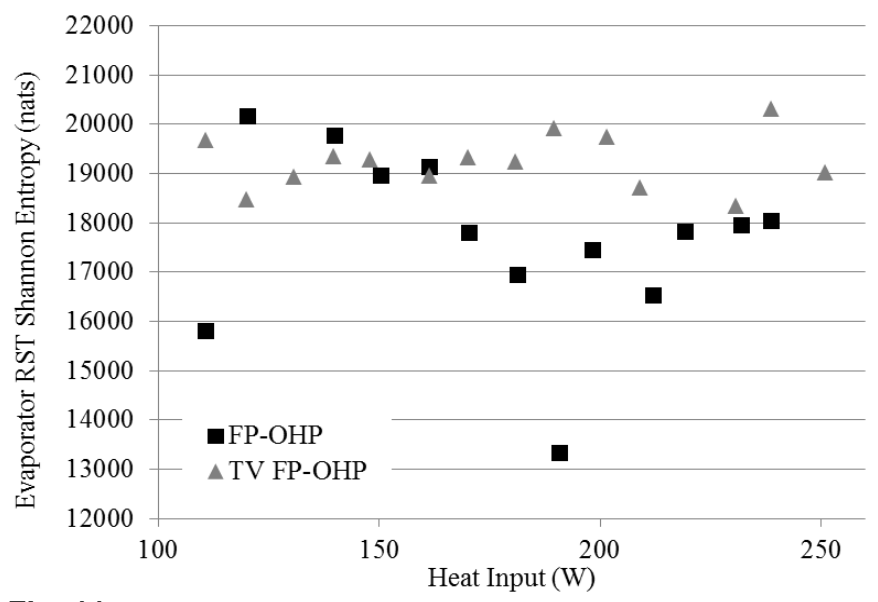

Fig. 11 Shannon entropy in evaporator RST vs. heat input for regular FP-OHP and TV FP-OHP

\subsection{Kurtosis}

The unbiased, sample kurtosis in evaporator RST for the regular FPOHP and TV FP-OHP at various heat inputs is shown in Fig. 12.

As shown in Fig. 12, the TV FP-OHP provided evaporator RST with higher kurtosis than that of the regular FP-OHP at almost all heat inputs investigated. This indicates that the evaporator RST for the TV FP-OHP provided for probability distributions with more 'peakedness', meaning that the PDF for evaporator RST was more centered near zero than that of the regular FP-OHP. The higher kurtosis in the evaporator RST for the TV FP-OHP, along with the higher Shannon entropy, may suggest the stronger existence of bulk fluid circulation within the evaporator. 


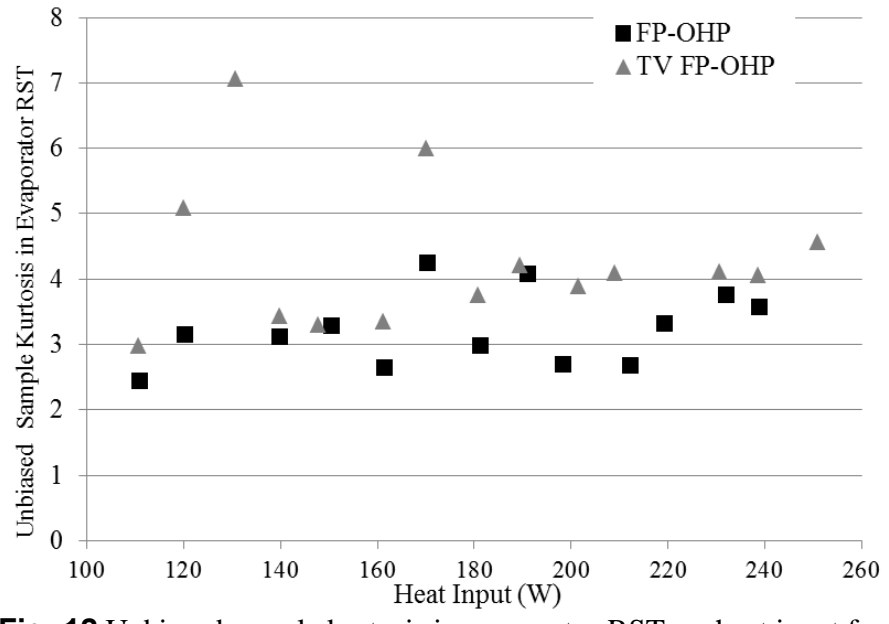

Fig. 12 Unbiased sample kurtosis in evaporator RST vs. heat input for regular FP-OHP and TV FP-OHP

\section{CONCLUSIONS}

The recorded temperature signals occurring on the wall of FP-OHPs either with or without Tesla-type check valves were analyzed using statistical methods and parameters. The main results are summarized as follows:

1) After OHP activation, the time-varying temperature signals can be modeled as emissions of a random variable, $\Omega_{\mathrm{q}}$, sampling from a heat-dependent, multi-Gaussian PDF, $\mathrm{f}_{\mathrm{q}}$. Using the Expectation-Maximization algorithm, the PDF is well-approximated via the Gaussian mixture model (GMM).

2) The temperature signals must consist of multiple frequency modes since the oscillatory temperature signals were not sufficiently modeled with a unimodal Gaussian PDF.

3) The $f_{q}$ for condenser RST was more sensitive to heat input and generally had less mixture components relative to the evaporator RST.

4) The PDF parameters, including the number of mixture components, of each GMM were found to vary with heat input for both heat pipes investigated.

5) Variance in the number of mixture components over the investigated heating history was less for the FP-OHP with Tesla-type check valves (TV FP-OHP).

6) The evaporator and condenser temperature signals were moderately, but not perfectly, correlated with each other as evidenced by the Pearson correlation coefficient having a magnitude between 0.6 and 0.8 . The TV FP-OHP provided for more correlated evaporator/condenser temperature signals.

7) At high heat inputs, the TV FP-OHP's evaporator temperature signal provided for higher Shannon entropy and consistently provided a PDF with higher kurtosis.

Using a variety of statistical parameters, it was determined that the rectified flow pattern within the TV FP-OHP provides for an oscillatory temperature field with features not found in the FP-OHP with no check valves. Therefore, the aforementioned parameters may be used to thermally detect flow rectification within OHPs. These results further demonstrate the complex, pseudo-random operation of the oscillating heat pipe.

\section{ACKNOWLEDGEMENTS}

The work presented in this article was supported by the Office of Naval Research Grant No. N00014-06-1-1119, under the direction of Dr. Mark Spector.

\section{NOMENCLATURE}

\begin{tabular}{|c|c|}
\hline$A$ & area $\left(\mathrm{m}^{2}\right)$ \\
\hline$B I C$ & Bayesian information criterion \\
\hline$D$ & diameter $(m)$ \\
\hline$f$ & probability density function \\
\hline$g$ & local acceleration due to gravity $\left(\mathrm{m} / \mathrm{s}^{2}\right)$ \\
\hline H & Shannon entropy (nats) \\
\hline$I Q R$ & interquartile range $\left({ }^{\circ} \mathrm{C}\right)$ \\
\hline$M$ & number of mixture components \\
\hline$n$ & sample size of one temperature signal \\
\hline$N$ & sample size of RST \\
\hline$P$ & probability \\
\hline$r$ & Pearson product-moment correlation coefficient \\
\hline$t$ & sample time $(\mathrm{s})$ \\
\hline$T$ & temperature $\left({ }^{\circ} \mathrm{C}\right)$ \\
\hline$T$ & temperature signal $\left({ }^{\circ} \mathrm{C}\right)$ \\
\hline$w$ & bin width $\left({ }^{\circ} \mathrm{C}\right)$ \\
\hline \multicolumn{2}{|c|}{ Greek Symbols } \\
\hline$\gamma$ & surface tension $(\mathrm{N} / \mathrm{m})$ \\
\hline$K$ & unbiased sample kurtosis \\
\hline$\lambda$ & estimated parameter matrix \\
\hline$\Lambda$ & number of GMM parameters \\
\hline$\hat{\mu}$ & unbiased estimator for the mean $\left({ }^{\circ} \mathrm{C}\right)$ \\
\hline$\Omega$ & regional/shifted temperature random variable $\left({ }^{\circ} \mathrm{C}\right)$ \\
\hline$\pi$ & array of mixture component weights \\
\hline$\rho$ & density $\left(\mathrm{kg} / \mathrm{m}^{3}\right)$ \\
\hline$\hat{\sigma}_{\mathrm{k}}$ & unbiased estimator for standard deviation $\left({ }^{\circ} \mathrm{C}\right)$ \\
\hline$\theta$ & negative of the log-likelihood function \\
\hline \multicolumn{2}{|c|}{ Subscripts } \\
\hline$c$ & condenser \\
\hline$e$ & evaporator \\
\hline$H$ & hydraulic \\
\hline hist & histogram \\
\hline$i$ & data sample index \\
\hline$j$ & thermocouple location index \\
\hline$k$ & mixture component index \\
\hline$l$ & liquid \\
\hline $\max$ & maximum \\
\hline $\min$ & minimum \\
\hline$R$ & regional \\
\hline$q$ & heat input \\
\hline$S$ & shifted \\
\hline$t / c$ & thermocouple \\
\hline$v$ & vapor \\
\hline
\end{tabular}

\section{REFERENCES}

Akachi, H., 1990, “Structure of a Heat Pipe,” U.S. Patent \#4,921,041.

Bardell, R.L., 2000, "The Diodicity Mechanism of Tesla-Type NoMoving-Parts Valves," Ph. D. Dissertation, University of Washington.

Cai, Q., Chen, C.-L., and Asfia, J.F., 2006, “Operating Characteristic Investigations in Pulsating Heat Pipe," Journal of Heat Transfer, 128 (12), pp. 1329-1334.

http://dx.doi.org/10.1115/1.2349509

Chen, P.H., Lee, Y.W., and Chang, T.L., 2009, "Predicting Thermal Instability in a Closed Loop Pulsating Heat Pipe System," Applied Thermal Engineering, 29 (8-9), pp. 1566-1576. http://dx.doi.org/10.1016/j.applthermaleng.2008.07.007 
Dempster, A.P., Laird, N.M., and Rubin, D.B., 1977, "Maximum Likelihood from Incomplete Data via the EM Algorithm," Journal of the Royal Statistical Society. Series B, 39 (1), pp. 1-38.

Freedman, D., and Diaconis, P., 1981, "On the Histogram as a Density Estimator: L2 Theory," Probability Theory and Related Fields, 57 (4), pp. 453-476.

Hartigan, J.A., and Wong, M.A., 1979, "A K-Means Clustering Algorithm," Journal of the Royal Statistical Society. Series C, 28 (1), pp. 100-108.

Kammuang-Lue, N., Sakulchangsatjatai, P., Terdtoon, P., and Mook, D.J., 2009, "Correlation to Predict the Maximum Heat Flux of a Vertical Closed-Loop Pulsating Heat Pipe," Heat Transfer Engineering, 30 (12), pp. 961-972.

http://dx.doi.org/10.1080/01457630902837442

Khandekar, S., Dollinger, N., and Groll, M., 2003, "Understanding Operation Regimes of Closed Loop Pulsating Heat Pipes: An Experimental Study," Applied Thermal Engineering, 23 (6), pp. 707719.

http://dx.doi.org/10.1016/S1359-4311(02)00237-5

Khandekar, S., Gautam, A.P., and Sharma, P.K., 2009, "Multiple Quasi-Steady States in a Closed Loop Pulsating Heat Pipe," International Journal of Thermal Sciences, 48 (3), pp. 535-546.

http://dx.doi.org/10.1016/j.ijthermalsci.2008.04.004

Lee, Y.W., and Chang, T.L., 2009, “Application of NARX Neural Networks in Thermal Dynamics Identification of a Pulsating Heat Pipe," Energy Conversion and Management, 50 (4), pp. 1069-1078. http://dx.doi.org/10.1016/j.enconman.2008.12.008

Liu, S., Li, J., Dong, X., and Chen, H., 2007, "Experimental Study on Flow Patterns and Improved Configurations for Pulsating Heat Pipes," Journal of Thermal Science, 16 (1), pp. 56-62.

http://dx.doi.org/10.1007/s11630-007-0056-8

Lloyd, S.P., 1982, "Least Square Quantization in PCM," IEEE Transactions on Information Theory," 28 (2), pp. 129-137.

http://dx.doi.org/10.1109/TIT.1982.1056489

Qu, J., Wu, H., Cheng, P., and Wang, X., 2009, "Non-Linear Analyses of Temperature Oscillations in a Closed-Loop Pulsating Heat Pipe," Int. Journal of Heat and Mass Transfer, 52, 2009, pp. 3481-3489.

http://dx.doi.org/10.1016/j.ijheatmasstransfer.2009.03.012

Rittidech, S., Pipatpaiboon, N., and Terdtoon, P., 2007, "Heat-Transfer Characteristics of a Closed-Loop Oscillating Heat-Pipe with Check Valves," Applied Energy, 84 (5), pp. 565-577.

http://dx.doi.org/10.1016/j.apenergy.2006.09.010

Schwarz, G., 1978, "Estimating the Dimension of a Model," The Annals of Statistics, 6 (2), pp. 461-464.

http://dx.doi.org/10.1214/aos/1176344136
Shannon, C.E., 1948, "A Mathematical Theory of Communication," Bell System Technical Journal, 27, pp. 379-423, 623-656.

Song, Y., and Xu, J., "Chaotic Behavior of Pulsating Heat Pipes," International Journal of Heat and Mass Transfer, 52, 2009, pp. 29322941.

http://dx.doi.org/10.1016/j.ijheatmasstransfer.2009.02.030

Tesla, N., 1920, “Valvular conduit,” U.S. Patent \#1,329,559.

Thompson, S.M., and Ma, H.B., 2010, "Effect of Localized Heating on Three-Dimensional Flat-Plate Oscillating Heat Pipe," Advances in Mechanical Engineering, Article ID 465153, pp. 1-10.

Thompson, S.M., Ma, H.B., Winholtz, R.A., and Wilson, C., 2009, "Experimental Investigation of a Miniature Three-Dimensional FlatPlate Oscillating Heat Pipe," Journal of Heat Transfer, 131 (4), 0432101-0432109.

http://dx.doi.org/10.1115/1.3072953

Thompson, S.M., Cheng, P, and Ma, H.B, 2011, “An Experimental Investigation of a Three-Dimensional Flat-Plate Oscillating Heat Pipe with Staggered Microchannels," International Journal of Heat and Mass Transfer, 54 (17-18), pp. 3951-3959.

http://dx.doi.org/10.1016/j.ijheatmasstransfer.2011.04.030

Thompson, S.M., Ma, H.B., and Wilson, C., 2011, "Investigation of a Flat-Plate Oscillating Heat Pipe with Tesla-Type Check Valves," Experimental Thermal and Fluid Science, 35 (7), pp. 1265-1273. http://dx.doi.org/10.1016/j.expthermflusci.2011.04.014

Tong, B.Y., Wong, T.N., and Ooi, K.T., 2001, "Closed-Loop Pulsating Heat Pipe,” Applied Thermal Engineering, 21 (18), pp. 1845-1862. http://dx.doi.org/10.1016/S1359-4311(01)00063-1

Wilson, C., Borgmeyer, B., Winholtz, R.A., Ma, H.B., Jacobson, D.L., Hussey, D.S., and Arif, M., "Visual Observation of Oscillating Heat Pipes Using Neutron Radiography," Journal of Thermophysics and Heat Transfer, 22 (3), 2008, pp. 366-372.

http://dx.doi.org/10.2514/1.33758

Xu, J.L., Li, Y.X., and Wong, T.N., 2005, "High Speed Flow Visualization of a Closed Loop Pulsating Heat Pipe," International Journal of Heat and Mass Transfer, 48 (16), pp. 3338-3351. http://dx.doi.org/10.1016/j.ijheatmasstransfer.2005.02.034

Xu, J.L., and Zhang, X.M., 2005, "Start-up and Steady Thermal Oscillation of a Pulsating Heat Pipe," Heat and Mass Transfer, 41 (8), pp. 685-694.

http://dx.doi.org/10.1007/s00231-004-0535-3

Yang, H., Khandekar, S., and Groll, M., 2008, "Operation Limit of Closed Loop Pulsating Heat Pipes," Applied Thermal Engineering, 28 (1), pp. 49-59.

http://dx.doi.org/10.1016/j.applthermaleng.2007.01.033 